\title{
Effects of fish oil supplementation on the fatty acid profile in erythrocyte membrane and plasma phospholipids of pregnant women and their offspring: a randomised controlled trial
}

\author{
M. Victoria Escolano-Margarit ${ }^{1}$, Cristina Campoy ${ }^{1 *}$, M. Carmen Ramírez-Tortosa ${ }^{2}$, Hans Demmelmair ${ }^{3}$, \\ M. Teresa Miranda ${ }^{4}$, Angel Gil ${ }^{2}$, Tamás Decsi ${ }^{5}$ and Berthold V. Koletzko ${ }^{3}$ \\ ${ }^{1}$ Department of Pediatrics, School of Medicine, University of Granada, Avenida de Madrid 11, 18012 Granada, Spain \\ ${ }^{2}$ Department of Biochemistry and Molecular Biology, University of Granada, Campus Universitario de Cartuja, 18071 \\ Granada, Spain \\ ${ }^{3}$ Hauner Children's Hospital, University of Munich Medical Centre, Lindwurmstrasse 4, D-80337 Munich, Germany \\ ${ }^{4}$ Department of Biostatistics, University of Granada, Avenida de Madrid 11, 18012 Granada, Spain \\ ${ }^{5}$ Department of Paediatrics, University of Pécs, H-7623 Pécs, Hungary \\ (Submitted 30 March 2012 - Final revision received 19 July 2012 - Accepted 20 July 2012 - First published online 5 September 2012)
}

\section{Abstract}

We aimed to investigate the effects of fish oil (FO) supplementation to pregnant women on the maternal and fetal fatty acid profile in plasma and erythrocyte phospholipids (PL) and to identify the best compartment for the assessment of fatty acid status. A multi-centre, double-blind, controlled trial was conducted. Healthy pregnant women from three European centres were randomly assigned to receive from week 20 of gestation until delivery a daily dietary supplement with either FO (500 mg DHA + 150 mg EPA), $400 \mu \mathrm{g} 5$-methyltetrahydrofolate, both or placebo. Fatty acids in plasma and erythrocyte PL were determined in maternal blood (week 20, week 30 of pregnancy and delivery) and in cord blood (delivery). FO supplementation increased DHA levels in maternal and cord plasma and erythrocyte PL. Higher percentage changes were observed in erythrocyte PL than in plasma PL. There were significant correlations between plasma and erythrocyte fatty acid levels in maternal and cord blood. Significant correlations between maternal and cord fatty acid levels at delivery in plasma and erythrocytes were also observed; however, correlation coefficients were higher for erythrocyte phophatidylethanolamine. FO supplementation increases maternal and fetal DHA status. Both plasma and erythrocytes appear to be suitable to evaluate the fatty acid status of mothers but erythrocytes seem to be a more reliable marker in neonates.

Key words: DHA: Long-chain PUFA: Pregnancy: Folate: Erythrocyte membrane phospholipids: Plasma phospholipids

Fetal metabolism and development depend on nutrients crossing the placenta and, therefore, on maternal nutritional status and diet during pregnancy. The essential fatty acids and their derivatives, long-chain PUFA (LC-PUFA), are essential constituents of membranes, especially in the brain and retina ${ }^{(1)}$, and must be provided to the fetus.

Given the importance of LC-PUFA in fetal development, in recent years, growing interest has been given to maternal fatty acid status. It is well known from interventional studies that LC-PUFA supplementation to pregnant women significantly increases LC-PUFA concentrations in both plasma and erythrocyte phospholipids (PL) in the mother as well as in the fetus ${ }^{(2-7)}$. Few studies have assessed the effects of supplementation on plasma and erythrocyte PL in the same population $^{(8,9)}$. It is generally accepted that LC-PUFA in plasma PL reflect tissue LC-PUFA status. Recent discussion has centred on whether erythrocyte membrane PL might be preferred to assess fatty acid status ${ }^{(10,11)}$. In addition, a positive correlation between folate and DHA levels has been reported in human subjects ${ }^{(12)}$. Although the detailed mechanism remains unclear, the effect of folate supplementation on fatty acid status might be attributable to the increased methionine availability present after supplementation, which stimulates the metabolism of essential fatty acids ${ }^{(13,14)}$.

The present study was performed to investigate the effects of supplementation with fish oil (FO) and/or 5-methyltetrahydrofolate (5-MTHF) from week 20 of gestation until delivery on the fatty acid profile of plasma and erythrocyte PL of

Abbreviations: 5-MTHF, 5-methyltetrahydrofolate; AA, arachidonic acid; FO, fish oil; LC-PUFA, long-chain PUFA; PC, phosphatidylcholine; PE, phosphatidylethanolamine; PL, phospholipids.

*Corresponding author: Professor C. Campoy, fax +34 958240740, emails ccampoy@ugr.es; ccfolgoso@gmail.com 
pregnant women during the course of pregnancy and fetal LC-PUFA status. We also aimed to elucidate which is the best compartment for the assessment of the fatty acid status of pregnant women and their neonates.

\section{Methods \\ Study design}

Information regarding study design, recruitment, inclusion criteria, dietary intervention, and collection of data and biological material has been reported elsewhere ${ }^{(15)}$. Briefly, a multicentre, randomised, double-blind controlled trial was conducted. A total of 315 healthy pregnant women were recruited before gestation week 20 at three different European centres (Ludwig Maximilians University, Munich (Germany), the University of Granada (Spain) and the University of Pecs (Hungary)). Women who consumed FO supplements since the beginning of pregnancy or vitamin $\mathrm{B}_{12}$ or folate supplements after gestation week 16 were excluded from the study. They were randomly assigned to four different groups and received daily a dietary supplement, from gestation week 20 until delivery, consisting of FO (500 mg DHA + $150 \mathrm{mg}$ EPA; Pronova Biocare), $400 \mu \mathrm{g}$ 5-MTHF (BASF), both or placebo together with vitamins and minerals in amounts meeting the recommended intakes during the second half of pregnancy for European women. The mothers were physically examined at the 20th and 30th week of gestation and data on the course of pregnancy and dietary intake were collected by means of standardised questionnaires. At delivery, information about delivery and data on the newborn were collected. At the 20th and 30th week of gestation, $10 \mathrm{ml}$ of maternal venous blood were collected into EDTA, and at delivery, $12 \mathrm{ml}$ of maternal venous blood as well as $12 \mathrm{ml}$ of venous cord blood were collected. The main outcome variables were fatty acid relative concentrations (wt\%) in plasma and erythrocyte membrane PL of cord and maternal blood.

The study protocol was approved by the Medical Ethics Committees of all centres participating in the study. Written informed consent was obtained from all participants. The present trial was registered at www.clinicaltrials.gov (registration no. NCT01180933).

\section{Biochemical analyses}

Sample collection procedure. At the 20th week (before supplementations) and at the 30th week of gestation, $10 \mathrm{ml}$ of maternal blood were collected into EDTA-containing tubes by venepuncture after an overnight fast. At delivery, $12 \mathrm{ml}$ of maternal blood were obtained as described for the samples at the 20th and 30th week of pregnancy. Further, $12 \mathrm{ml}$ of placental venous cord blood samples were collected into EDTA-containing tubes at delivery after clamping of the umbilical cord and before parturition of the placenta by puncture and aspiration from a long cord segment. Blood was centrifuged at $3500 \mathrm{rpm}$ for $10 \mathrm{~min}$ at room temperature within $2 \mathrm{~h}$. Plasma samples were frozen in liquid $\mathrm{N}_{2}$ at $-196^{\circ} \mathrm{C}$ in 100 and $200 \mu \mathrm{l}$ aliquots and stored at $-80^{\circ} \mathrm{C}$ until fatty acid analyses. Blood cells were washed three times in isotonic $\mathrm{NaCl}$ solution. The last sediment was haemolysed in $1 \mathrm{ml}$ of distilled water for $20 \mathrm{~min}$ at room temperature, and then $2 \mathrm{ml}$ ice-cold isopropylic alcohol with $0.5 \%$ butylated hydroxytoluene were added as an antioxidant and the samples were stored at $-80^{\circ} \mathrm{C}$ until further analyses.

Analyses of fatty acids in maternal and umbilical cord plasma and erythrocyte phospholipids. Total lipid extraction from plasma was performed according to the method of Kolarovic \& Fournier ${ }^{(16)}$. Briefly, $0.5 \mathrm{ml}$ plasma plus $0.5 \mathrm{ml}$ water were vortexed for $30 \mathrm{~s}$ with $100 \mu \mathrm{l}$ of internal standard (0.857g diheptadecanoyl phosphatidylcholine (PC)/1 dissolved in chloroform). Then, lipids were extracted four times, initially with $n$-hexane-2-propanolol (3:2, v/v) containing $25 \mathrm{mg} / \mathrm{l}$ of butylated hydroxytoluene as an antioxidant and then three times with pure hexane. The pooled extracts were dried under vacuum and dissolved in $200 \mu$ l hexane-methyltert-butyl-diethyl ether-acetic acid (100:3:0·3, by vol.) and PL were isolated by liquid chromatography using aminopropyl columns (Sep Pak Cartridges; Waters) as described by Agren et al. ${ }^{(17)}$.

Lipids from erythrocytes were extracted by adding $3 \mathrm{ml}$ chloroform and two internal standards (phosphatidylethanolamine (PE)-heptadecanoate and PC-pentadecanoate esters dissolved in methanol). The mixture was shaken on a vortex and the lower layer was aspirated and evaporated under a $\mathrm{N}_{2}$ stream. The dry lipid extract was resolved in chloroform and added to silica gel plates (Merck 60, $10 \times 20 \mathrm{~cm}$ ). The runner solvent for the first run was hexane-diethyl ether-chloroform-acetic acid (21:6:3:1, by vol.). The plate was dried under a hood at ambient temperature and ran again with chloroform-methanol-water (65:25:4, by vol.). For positioning PC and PE ester bands, the proper oleates were ran in parallel in every plate. The bands were stained with dichlorofluorescein, visualised under UV light and scrapped for transmethylation.

Fatty acid methyl esters from individual fractions were obtained by reaction with $3 \mathrm{M}-\mathrm{HCl}-$ methanol at $84^{\circ} \mathrm{C}$ for $40 \mathrm{~min}$ according to the method of Lepage \& Roy ${ }^{(18)}$.

The quantification of fatty acid methyl esters from plasma PL was performed by GC using a gas chromatograph (HP5890 Series II; Hewlett Packard) equipped with a flame ionisation detector. Chromatography was performed using a $60 \mathrm{~m}$-long capillary column with a $0.32 \mathrm{~mm}$ internal diameter and $0.20 \mu \mathrm{m}$ thickness and impregnated with SP-2330 FS (Supelco). The injector and the detector were maintained at 250 and $275^{\circ} \mathrm{C}$, respectively. $\mathrm{N}_{2}$ was used as the carrier gas and the split ratio was 29:1. Fatty acid methyl esters were identified by comparison of retention times with those of known standards.

Analysis of fatty acid methyl esters from erythrocyte PE and PC was performed by high-resolution capillary GLC (model 9001 GC; Finnigan/Tremetrics) with a split injection, an automatic sampler (A200SE; CTC Analytic) and a flame ionisation detector with a DB-23 cyanopropyl column of $60 \mathrm{~m}$ length ( $\mathrm{J} \& \mathrm{~W}$ Scientific). The conditions during the analysis were as follows: temperature of the injector at $80^{\circ} \mathrm{C}$ for $0 \cdot 1 \mathrm{~min}$, temperature increase by $180^{\circ} \mathrm{C} / \mathrm{min}$ up to $280^{\circ} \mathrm{C}$, temperature of 
the column area at $60^{\circ} \mathrm{C}$ for $0 \cdot 2 \mathrm{~min}$, temperature increase by $40^{\circ} \mathrm{C} / \mathrm{min}$ up to $180^{\circ} \mathrm{C}$, a $5 \mathrm{~min}$ hold period, temperature increase by $1.5^{\circ} \mathrm{C} / \mathrm{min}$ up to $200^{\circ} \mathrm{C}$, an $8.5 \mathrm{~min}$ hold period, temperature increase by $40^{\circ} \mathrm{C} / \mathrm{min}$ up to $240^{\circ} \mathrm{C}$ and a $13 \mathrm{~min}$ hold period. The constant linear velocity was $0.3 \mathrm{~m} / \mathrm{s}$ (referred to $100^{\circ} \mathrm{C}$ ). For identification of sample peaks, we used two commercially available fatty acid methyl ester calibration mixtures (Supelco 37 FAME mix and NU-CKECK GLC reference 463) containing the fatty acids measured in the present study.

Results are expressed as percentages by weight (wt \%) of total detected fatty acids with $14-24 \mathrm{C}$ atoms

\section{Statistical analyses}

The Kolmogorov-Smirnov or Shapiro-Wilk test was used to test the normality of variables. Baseline characteristics were compared among the intervention groups by using the Kruskal-Wallis test for continuous data and the $\chi^{2}$ test for ordinal data. A three-factor repeated-measures ANOVA with the intervention group as the between-subject factor and pregnancy time points (gestation week 20, gestation week 30 and delivery) as within-subject factors was performed to compare the effects of supplementation with time. Differences in cord blood fatty acid levels were assessed separately by using ANOVA for normally distributed variables and the Kruskal-Wallis test for not normally distributed variables. If significant effects were observed, multiple comparisons with Bonferroni correction were performed.

Spearman's correlations $(\rho)$ were performed to determine the correlations between plasma and erythrocyte relative levels of fatty acids and between maternal fatty acid status at delivery and neonatal fatty acid status at birth. $P$ values less than 0.05 were considered as significant.

Statistical analyses were performed using SPSS statistical software (version 15.0; SPSS Institute).

\section{Results}

\section{Sample description}

Information regarding sample description, compliance, dropouts and baseline characteristics of study participants has been reported previously ${ }^{(15)}$. Briefly, 315 healthy women with single pregnancies were recruited before the 20th week of gestation. Of these, four did not fulfil the inclusion criteria and were therefore excluded. Furthermore, forty-one women did not complete the study; the main reasons for dropping out were non-compliance, relocation and bad taste of the supplement (FO: 10·4\%; FO + 5-MTHF: 16.9\%; 5-MTHF: 16.6\%;

Table 1. Baseline characteristics of the study population after randomisation to intervention groups

(Mean values and standard deviations*; number of subjects and percentages $\dagger$ )

\begin{tabular}{|c|c|c|c|c|c|c|c|c|c|}
\hline & \multicolumn{2}{|c|}{$\mathrm{FO}(n 69)$} & \multicolumn{2}{|c|}{$\begin{array}{c}\mathrm{FO}+ \\
5-\mathrm{MTHF} \\
(n 64)\end{array}$} & \multicolumn{2}{|c|}{$\begin{array}{c}\text { 5-MTHF } \\
(n \text { 65) }\end{array}$} & \multicolumn{2}{|c|}{$\begin{array}{c}\text { Placebo } \\
(n 72)\end{array}$} & \multirow[b]{2}{*}{$P$} \\
\hline & $n$ & $\%$ & $n$ & $\%$ & $n$ & $\%$ & $n$ & $\%$ & \\
\hline Age (years) & & & & & & & & & NS \\
\hline Mean & \multicolumn{2}{|c|}{31} & \multicolumn{2}{|c|}{31} & \multicolumn{2}{|c|}{30} & \multicolumn{2}{|c|}{31} & \\
\hline SD & \multirow{2}{*}{\multicolumn{2}{|c|}{7}} & \multicolumn{2}{|c|}{8} & \multicolumn{2}{|c|}{10} & \multicolumn{2}{|c|}{6} & \\
\hline Parity & & & & & & & & & NS \\
\hline 0 & 40 & $58 \cdot 0$ & 30 & $46 \cdot 9$ & 32 & $49 \cdot 2$ & 37 & 51.4 & \\
\hline$\geq 1$ & 29 & $42 \cdot 0$ & 34 & $53 \cdot 1$ & 33 & $50 \cdot 8$ & 35 & $48 \cdot 6$ & \\
\hline Smoking & 10 & 14.5 & 11 & $17 \cdot 2$ & 11 & $16 \cdot 9$ & 6 & $8 \cdot 3$ & NS \\
\hline Gestational age (weeks) & & & & & & & & & NS \\
\hline Mean & \multicolumn{2}{|c|}{39} & \multicolumn{2}{|c|}{39} & \multicolumn{2}{|c|}{38} & \multicolumn{2}{|c|}{39} & \\
\hline SD & \multicolumn{2}{|c|}{2} & \multicolumn{2}{|c|}{2} & \multicolumn{2}{|c|}{3} & \multicolumn{2}{|c|}{2} & \\
\hline Ethnicity (\%) & & & & & & & & & NS \\
\hline Caucasian & 69 & 100 & 63 & 98.4 & 64 & 98.5 & 70 & $97 \cdot 2$ & \\
\hline Others & 0 & 0 & 1 & 1.6 & 1 & 1.5 & 2 & $2 \cdot 8$ & \\
\hline Residence area (\%) & & & & & & & & & NS \\
\hline City area & 32 & $46 \cdot 4$ & 29 & $45 \cdot 3$ & 32 & $49 \cdot 2$ & 37 & 51.4 & \\
\hline Farm area & 37 & $53 \cdot 6$ & 35 & $54 \cdot 7$ & 33 & $50 \cdot 8$ & 35 & $48 \cdot 6$ & \\
\hline Gravidity risk (20 weeks) & & & & & & & & & NS \\
\hline No risk factor & 19 & $27 \cdot 5$ & 20 & $31 \cdot 2$ & 18 & $27 \cdot 7$ & 28 & 38.9 & \\
\hline$\geq 1$ risk factor & 50 & 72.5 & 44 & 68.8 & 47 & $72 \cdot 3$ & 44 & $61 \cdot 1$ & \\
\hline Delivery risk & & & & & & & & & NS \\
\hline No risk factor & 30 & 43.5 & 35 & $54 \cdot 7$ & 35 & $53 \cdot 8$ & 45 & $62 \cdot 5$ & \\
\hline$\geq 1$ risk factor & 39 & 56.5 & 29 & $45 \cdot 3$ & 30 & $46 \cdot 2$ & 27 & 37.5 & \\
\hline \multicolumn{10}{|l|}{$\mathrm{BMI}\left(\mathrm{kg} / \mathrm{m}^{2}\right)$} \\
\hline 20 weeks & \multirow{2}{*}{\multicolumn{2}{|c|}{$26 \cdot 0$}} & & & & & & & NS \\
\hline Mean & & & \multicolumn{2}{|c|}{$25 \cdot 2$} & \multicolumn{2}{|c|}{25.2} & \multicolumn{2}{|c|}{24.9} & \\
\hline SD & \multicolumn{2}{|c|}{4.0} & \multicolumn{2}{|c|}{$2 \cdot 9$} & \multicolumn{2}{|c|}{$4 \cdot 1$} & & & \\
\hline 30 weeks & & & & & & & & & NS \\
\hline Mean & & & & & & & & & \\
\hline SD & & & & & & & & & \\
\hline
\end{tabular}

FO, fish oil; 5-MTHF, 5-methyltetrahydrofolate.

* For continuous variables.

†For categorical variables. 
placebo: $10 \% ; P=0 \cdot 47$ ). Compliance was good; $89.5 \%$ of the subjects in the second trimester of pregnancy and $87.4 \%$ in the third missed less than $5 \mathrm{~d}$ supplementation. Overall, 270 maternal blood samples could be drawn at the 30th week of pregnancy and 243 at delivery. The available cord blood samples for analyses were 220.

The baseline characteristics of the study participants are shown in Table 1. Maternal clinical or sociodemographic characteristics were similarly distributed in the four intervention groups $(P>0.05)$. There were no significant differences with respect to the course of pregnancy or delivery complications between the groups $(P>0 \cdot 05)$. The habitual basal dietary intake of energy, fatty acids and nutrients of the participating mothers was similar in the four groups throughout the study ( $P>0.05)$ except for the supplementation (Table S1, available online).

\section{Fatty acid patterns in maternal plasma and erythrocyte phospholipids during pregnancy and in umbilical plasma and erythrocyte phospholipids at delivery}

The mean values of fatty acids in maternal plasma and erythrocyte PL during pregnancy and in umbilical plasma and erythrocyte PL at delivery are shown in Tables 2-4.

DHA in maternal plasma PL and erythrocyte PC significantly increased during the second half of pregnancy in the FO and $\mathrm{FO}+5$-MTHF groups $(P<0 \cdot 001)$ compared with the placebo and 5-MTHF groups. DHA in maternal erythrocyte PE significantly increased during the second half of pregnancy in all

Table 2. Relative fatty acid composition (wt\%) of maternal and neonatal plasma phospholipids (Mean values and standard deviations)

\begin{tabular}{|c|c|c|c|c|c|c|c|c|c|}
\hline & & \multicolumn{2}{|c|}{ Week 20} & \multicolumn{2}{|c|}{ Week 30} & \multicolumn{2}{|c|}{ Delivery } & \multicolumn{2}{|c|}{ Newborn } \\
\hline & & Mean & SD & Mean & SD & Mean & SD & Mean & SD \\
\hline \multirow[t]{4}{*}{$18: 2 n-6$} & $\mathrm{FO}^{*}$ & $21 \cdot 22$ & $2 \cdot 79$ & $20 \cdot 50$ & 2.95 & 19.98 & 3.01 & 8.02 & 1.91 \\
\hline & $\mathrm{FO}+5-\mathrm{MTHF} \dagger$ & $21.69^{A}$ & 2.43 & $20 \cdot 71^{\mathrm{B}}$ & $2 \cdot 34$ & $19 \cdot 99^{\mathrm{B}}$ & 2.92 & 7.86 & 1.21 \\
\hline & 5-MTHF & 21.55 & 2.53 & $21 \cdot 38$ & $2 \cdot 18$ & $20 \cdot 82$ & 2.39 & 7.41 & 1.02 \\
\hline & Placebo§ & $21 \cdot 33$ & $2 \cdot 71$ & 21.43 & $2 \cdot 25$ & $20 \cdot 77$ & $2 \cdot 83$ & $7 \cdot 34$ & 1.06 \\
\hline \multirow[t]{4}{*}{$18: 3 n-3$} & FO & 0.04 & $0 \cdot 11$ & 0.07 & 0.12 & 0.05 & 0.11 & $\|$ & $\|$ \\
\hline & $\mathrm{FO}+5-\mathrm{MTHF}$ & 0.04 & 0.11 & 0.04 & 0.11 & 0.06 & 0.13 & $\|$ & $\|$ \\
\hline & 5-MTHF & $0.02^{\mathrm{B}}$ & 0.09 & $0.08^{\mathrm{A}}$ & 0.13 & $0.11^{\mathrm{A}}$ & 0.14 & $\|$ & $\|$ \\
\hline & Placebo & 0.04 & $0 \cdot 12$ & 0.09 & 0.13 & 0.05 & 0.11 & $\|$ & $\|$ \\
\hline \multirow[t]{4}{*}{$20: 4 n-6$} & FO & $10 \cdot 41^{\mathrm{A}}$ & 1.82 & $8 \cdot 88^{\mathrm{B}}$ & 1.47 & $8.93^{\mathrm{B}}$ & 1.90 & $16 \cdot 47^{\mathrm{a}, \mathrm{b}}$ & $2 \cdot 33$ \\
\hline & $\mathrm{FO}+5-\mathrm{MTHF}$ & $10 \cdot 40^{\mathrm{A}}$ & 1.59 & $8 \cdot 89^{\mathrm{B}}$ & 1.27 & $8.94^{\mathrm{B}}$ & 1.86 & $16 \cdot 39^{\mathrm{b}}$ & 2.04 \\
\hline & 5-MTHF & $10 \cdot 24^{\mathrm{A}}$ & $2 \cdot 12$ & $9 \cdot 20^{\mathrm{B}}$ & 1.73 & $9 \cdot 08^{\mathrm{B}}$ & 1.77 & $17 \cdot 38^{\mathrm{a}}$ & 1.80 \\
\hline & Placebo & $10 \cdot 18^{\mathrm{A}}$ & 1.47 & $9 \cdot 28^{\mathrm{B}}$ & 1.56 & $9 \cdot 46^{\mathrm{B}}$ & 1.63 & $17 \cdot 58^{\mathrm{a}}$ & 1.88 \\
\hline \multirow[t]{4}{*}{$22: 6 n-3$} & FO & $4.43^{\mathrm{B}}$ & 1.05 & $5 \cdot 87^{\mathrm{a}, \mathrm{A}}$ & 1.61 & $5 \cdot 69^{\mathrm{a}, \mathrm{A}}$ & 1.42 & $7 \cdot 53^{\mathrm{a}}$ & 1.69 \\
\hline & $\mathrm{FO}+5-\mathrm{MTHF}$ & $4.54^{\mathrm{C}}$ & 1.09 & $6 \cdot 26^{\mathrm{a}, \mathrm{A}}$ & 1.17 & $5.84^{\mathrm{a}, \mathrm{B}}$ & 1.30 & $7.09^{a, b}$ & 1.83 \\
\hline & 5-MTHF & 4.46 & 0.86 & $4.43^{b}$ & 0.81 & $4 \cdot 31^{b}$ & 1.20 & $6.06^{\mathrm{C}}$ & 1.62 \\
\hline & Placebo & $4 \cdot 66^{\mathrm{A}}$ & $1 \cdot 14$ & $4 \cdot 19^{\mathrm{b}, \mathrm{B}}$ & 0.99 & $4 \cdot 20^{\mathrm{b}, \mathrm{B}}$ & 1.24 & $6 \cdot 53^{\mathrm{b}, \mathrm{c}}$ & 1.62 \\
\hline \multirow[t]{4}{*}{$\Sigma S F A$} & FO & 43.75 & $2 \cdot 32$ & $43 \cdot 71$ & $1 \cdot 27$ & $44 \cdot 19$ & 1.21 & 48.95 & 1.71 \\
\hline & $\mathrm{FO}+5-\mathrm{MTHF}$ & $43 \cdot 76^{\mathrm{A}, \mathrm{B}}$ & $1 \cdot 13$ & $43 \cdot 44^{\mathrm{B}}$ & 1.22 & $44 \cdot 14^{\mathrm{A}}$ & 1.53 & $48 \cdot 75$ & 1.50 \\
\hline & 5-MTHF & 43.77 & 1.25 & 43.68 & 1.05 & 43.89 & 1.20 & $49 \cdot 30$ & 1.55 \\
\hline & Placebo & 43.68 & 1.49 & 43.94 & 0.90 & 44.09 & 1.35 & $49 \cdot 32$ & 2.08 \\
\hline \multirow[t]{4}{*}{ ¿MUFA } & $\mathrm{FO}$ & $13 \cdot 37^{\mathrm{B}}$ & 2.49 & $14.46^{\mathrm{A}, \mathrm{B}}$ & 1.69 & $14.80^{\mathrm{a}, \mathrm{b}, \mathrm{A}}$ & 1.73 & 12.58 & $2 \cdot 21$ \\
\hline & $\mathrm{FO}+5-\mathrm{MTHF}$ & $13 \cdot 20$ & $2 \cdot 34$ & $14 \cdot 21$ & 1.68 & $14.49^{b}$ & 1.79 & 13.58 & $2 \cdot 60$ \\
\hline & 5-MTHF & $13 \cdot 62^{B}$ & $2 \cdot 34$ & $15 \cdot 11^{\mathrm{A}}$ & 1.63 & $15 \cdot 31^{\mathrm{a}, \mathrm{A}}$ & 2.00 & $13 \cdot 12$ & 2.03 \\
\hline & Placebo & 13.49 & $2 \cdot 18$ & 14.71 & 1.60 & $14.98^{a, b}$ & 1.69 & $12 \cdot 55$ & 2.96 \\
\hline \multirow{4}{*}{ ¿PUFA } & $\mathrm{FO}$ & $41.92^{A}$ & $3 \cdot 20$ & $41.09^{\mathrm{a}, \mathrm{b}, \mathrm{A}}$ & 2.01 & $40 \cdot 26^{B}$ & 1.82 & $37 \cdot 61$ & $2 \cdot 35$ \\
\hline & $\mathrm{FO}+5-\mathrm{MTHF}$ & $42.50^{\mathrm{A}}$ & 2.92 & $41 \cdot 70^{\mathrm{a}, \mathrm{A}}$ & $1 \cdot 70$ & $40 \cdot 71^{\mathrm{B}}$ & 1.72 & 36.58 & 2.69 \\
\hline & 5-MTHF & $41.93^{\mathrm{A}}$ & 3.09 & $40 \cdot 43^{\mathrm{b}, \mathrm{B}}$ & 1.50 & $40.06^{\mathrm{B}}$ & 2.02 & 36.57 & 2.06 \\
\hline & Placebo & $42 \cdot 21^{\mathrm{A}}$ & 3.05 & $40 \cdot 60^{\mathrm{b}, \mathrm{A}, \mathrm{B}}$ & 1.60 & $40 \cdot 31^{B}$ & 1.91 & $36 \cdot 87$ & $2 \cdot 44$ \\
\hline \multirow[t]{4}{*}{ AA:DHA } & FO & $2 \cdot 47^{\mathrm{A}}$ & 0.69 & $1.63^{\mathrm{b}, \mathrm{B}}$ & 0.57 & $1.67^{\mathrm{b}, \mathrm{B}}$ & 0.57 & $2 \cdot 30^{\mathrm{b}}$ & 0.65 \\
\hline & $\mathrm{FO}+5-\mathrm{MTHF}$ & $2 \cdot 41^{\mathrm{A}}$ & 0.66 & $1.48^{\mathrm{b}, \mathrm{B}}$ & 0.39 & $1.61^{\mathrm{b}, \mathrm{C}}$ & 0.53 & $2 \cdot 46^{\mathrm{b}}$ & 0.71 \\
\hline & 5-MTHF & $2 \cdot 38^{A}$ & 0.65 & $2 \cdot 14^{\mathrm{a}, \mathrm{B}}$ & 0.58 & $2 \cdot 23^{\mathrm{a}, \mathrm{A}}$ & 0.66 & $3 \cdot 10^{\mathrm{a}}$ & 0.99 \\
\hline & Placebo & $2 \cdot 32$ & 0.65 & $2 \cdot 35^{\mathrm{a}}$ & 0.71 & $2 \cdot 40^{\mathrm{a}}$ & 0.69 & $2 \cdot 85^{a}$ & 0.76 \\
\hline \multirow[t]{4}{*}{$\sum n-6: \sum n-3$} & FO & $6 \cdot 85^{\mathrm{A}}$ & 1.66 & $4.65^{\mathrm{b}, \mathrm{B}}$ & 1.55 & $4 \cdot 90^{\mathrm{b}, \mathrm{B}}$ & 1.46 & $4 \cdot 13^{\mathrm{C}}$ & 0.99 \\
\hline & $\mathrm{FO}+5-\mathrm{MTHF}$ & $6 \cdot 78^{\mathrm{A}}$ & 1.80 & $4 \cdot 30^{\mathrm{b}, \mathrm{C}}$ & $1 \cdot 12$ & $4 \cdot 73^{\mathrm{b}, \mathrm{B}}$ & 1.34 & $4 \cdot 35^{\mathrm{b}, \mathrm{c}}$ & 1.26 \\
\hline & 5-MTHF & 6.74 & 1.39 & $6 \cdot 35^{\mathrm{a}}$ & 1.40 & $6 \cdot 40^{\mathrm{a}}$ & 1.65 & $5 \cdot 45^{\mathrm{a}}$ & 1.65 \\
\hline & Placebo & 6.58 & 1.98 & $6 \cdot 70^{\mathrm{a}}$ & 1.87 & $6 \cdot 86^{a}$ & 1.97 & $4 \cdot 91^{\mathrm{a}, \mathrm{b}}$ & 1.30 \\
\hline
\end{tabular}

FO, fish oil; 5-MTHF, 5-methyltetrahydrofoate; AA, arachidonic acid.

a,b,c Mean values within a column with unlike superscript letters were significantly different between the intervention groups $(P<0.05)$.

$\mathrm{A}, \mathrm{B}, \mathrm{C}$ Mean values within a row with unlike superscript letters were significantly different between the pregnancy time points $(P<0 \cdot 05)$.

${ }^{*} n 57$.

$\dagger n 51$.

$\ddagger n 56$.

$\S n 53$.

I| Concentration of $18: 3 n-3$ in cord plasma phospholipids was too low to be detected. 
Table 3. Relative fatty acid composition (wt \%) of maternal and neonatal erythrocyte phosphatidylcholine (Mean values and standard deviations)

\begin{tabular}{|c|c|c|c|c|c|c|c|c|c|}
\hline & & \multicolumn{2}{|c|}{ Week 20} & \multicolumn{2}{|c|}{ Week 30} & \multicolumn{2}{|c|}{ Delivery } & \multicolumn{2}{|c|}{ Newborn } \\
\hline & & Mean & SD & Mean & SD & Mean & SD & Mean & SD \\
\hline \multirow[t]{4}{*}{$18: 2 n-6$} & $\mathrm{FO}^{*}$ & $15 \cdot 67$ & $2 \cdot 68$ & $15 \cdot 35$ & 2.86 & $15 \cdot 70$ & $2 \cdot 72$ & 6.88 & $2 \cdot 32$ \\
\hline & $\mathrm{FO}+5-\mathrm{MTHF}+$ & $15 \cdot 62$ & $2 \cdot 60$ & 15.43 & 2.74 & $15 \cdot 48$ & 2.56 & $6 \cdot 72$ & $1 \cdot 17$ \\
\hline & 5-MTHF $\ddagger$ & $15 \cdot 55$ & $2 \cdot 08$ & 14.78 & 2.72 & 15.06 & $2 \cdot 77$ & 6.52 & 1.28 \\
\hline & Placebo§ & $15 \cdot 35$ & $2 \cdot 79$ & $15 \cdot 51$ & 2.48 & $15 \cdot 65$ & $2 \cdot 63$ & 5.95 & 1.01 \\
\hline \multirow[t]{4}{*}{$18: 3 n-3$} & FO & 0.11 & 0.09 & 0.15 & 0.13 & 0.16 & 0.13 & 0.04 & 0.04 \\
\hline & $\mathrm{FO}+5-\mathrm{MTHF}$ & 0.13 & $0 \cdot 10$ & 0.12 & 0.06 & 0.14 & 0.09 & 0.08 & 0.09 \\
\hline & 5-MTHF & 0.18 & 0.14 & 0.17 & 0.16 & 0.17 & 0.12 & 0.11 & 0.24 \\
\hline & Placebo & 0.14 & 0.13 & 0.14 & 0.11 & 0.13 & $0 \cdot 10$ & 0.04 & 0.04 \\
\hline \multirow[t]{4}{*}{$20: 4 n-6$} & FO & 6.64 & $3 \cdot 13$ & $7 \cdot 07^{\mathrm{b}}$ & 2.57 & 7.02 & $2 \cdot 56$ & $12 \cdot 11$ & 3.43 \\
\hline & $\mathrm{FO}+5-\mathrm{MTHF}$ & 7.34 & $2 \cdot 41$ & $6 \cdot 73^{b}$ & 2.48 & $7 \cdot 37$ & $2 \cdot 17$ & $12 \cdot 62$ & 2.51 \\
\hline & 5-MTHF & 7.40 & $2 \cdot 21$ & $7 \cdot 50^{\mathrm{b}}$ & 2.58 & 7.93 & 2.43 & 11.51 & 3.93 \\
\hline & Placebo & $7.56^{\mathrm{B}}$ & 2.41 & $8.69^{\mathrm{a}, \mathrm{A}}$ & $2 \cdot 20$ & $8 \cdot 13^{A, B}$ & 2.57 & $12 \cdot 88$ & 3.97 \\
\hline \multirow[t]{4}{*}{$22: 6 n-3$} & FO & $1.92^{\mathrm{B}}$ & 1.00 & $2 \cdot 95^{\mathrm{A}}$ & 1.57 & $3.41^{\mathrm{A}}$ & $1 \cdot 70$ & $3 \cdot 97^{\mathrm{a}}$ & 1.62 \\
\hline & $\mathrm{FO}+5-\mathrm{MTHF}$ & $2 \cdot 22^{\mathrm{B}}$ & $1 \cdot 13$ & $3 \cdot 19^{A}$ & 1.37 & $3.71^{\mathrm{A}}$ & 1.42 & $3.96^{\mathrm{a}}$ & 1.47 \\
\hline & 5-MTHF & $2 \cdot 41$ & 1.02 & $2 \cdot 43$ & $1 \cdot 12$ & 2.59 & $1 \cdot 11$ & $2 \cdot 71^{\mathrm{b}}$ & 1.27 \\
\hline & Placebo & $2 \cdot 41$ & $1 \cdot 15$ & $2 \cdot 75$ & 1.20 & 2.51 & 0.94 & $2 \cdot 82^{b}$ & 1.31 \\
\hline \multirow[t]{4}{*}{$\Sigma$ SFA } & FO & $53.92^{\mathrm{A}}$ & $4 \cdot 12$ & $52.95^{\mathrm{A}, \mathrm{B}}$ & 3.46 & $52 \cdot 62^{\mathrm{B}}$ & 3.54 & 54.98 & 4.45 \\
\hline & $\mathrm{FO}+5-\mathrm{MTHF}$ & 53.46 & 4.81 & 52.53 & $3 \cdot 28$ & 52.02 & 3.46 & 54.73 & 3.78 \\
\hline & 5-MTHF & $52 \cdot 38$ & 2.90 & $52 \cdot 28$ & 2.95 & $51 \cdot 75$ & $3 \cdot 19$ & $55 \cdot 68$ & 4.36 \\
\hline & Placebo & 52.99 & 3.89 & $51 \cdot 82$ & 3.57 & 51.95 & $3 \cdot 12$ & 55.92 & 4.52 \\
\hline \multirow[t]{4}{*}{ ¿MUFA } & FO & $16 \cdot 18$ & $2 \cdot 26$ & $15 \cdot 70$ & $2 \cdot 15$ & $15 \cdot 40$ & $2 \cdot 17$ & $13.95^{a, b}$ & 2.22 \\
\hline & $\mathrm{FO}+5-\mathrm{MTHF}$ & $15 \cdot 41^{\mathrm{A}, \mathrm{B}}$ & $2 \cdot 32$ & $15 \cdot 92^{\mathrm{A}}$ & 2.49 & $15 \cdot 26^{B}$ & 2.02 & $13 \cdot 54^{\mathrm{b}}$ & 1.81 \\
\hline & 5-MTHF & $16 \cdot 03$ & 1.67 & $16 \cdot 45$ & $2 \cdot 25$ & $15 \cdot 94$ & $2 \cdot 14$ & $14.91^{\mathrm{a}}$ & $2 \cdot 16$ \\
\hline & Placebo & $15 \cdot 52$ & $2 \cdot 13$ & $14 \cdot 84$ & 1.47 & 15.43 & 1.82 & $14 \cdot 20^{\mathrm{a}, \mathrm{b}}$ & $2 \cdot 32$ \\
\hline \multirow[t]{4}{*}{ ¿PUFA } & FO & $27 \cdot 55^{\mathrm{B}}$ & 4.66 & $29.03^{A, B}$ & 4.38 & $29.83^{\mathrm{A}}$ & 4.46 & $27 \cdot 88$ & $5 \cdot 30$ \\
\hline & $\mathrm{FO}+5-\mathrm{MTHF}$ & 28.69 & 4.02 & $29 \cdot 19$ & 4.43 & 30.44 & 3.54 & $28 \cdot 32$ & 4.04 \\
\hline & 5-MTHF & $29 \cdot 22$ & 3.43 & 28.69 & 4.96 & 29.84 & $4 \cdot 19$ & $25 \cdot 63$ & 5.52 \\
\hline & Placebo & $29.09^{B}$ & $4 \cdot 31$ & $31 \cdot 12^{\mathrm{A}}$ & 3.48 & $30 \cdot 27^{A, B}$ & 3.61 & $26 \cdot 19$ & $5 \cdot 64$ \\
\hline \multirow[t]{4}{*}{$A A: D H A$} & FO & $4.01^{\mathrm{A}}$ & 1.76 & $2 \cdot 84^{\mathrm{b}, \mathrm{c}, \mathrm{B}}$ & $1 \cdot 14$ & $2 \cdot 36^{\mathrm{b}, \mathrm{c}}$ & 0.82 & $3.44^{\mathrm{b}}$ & 1.44 \\
\hline & $\mathrm{FO}+5-\mathrm{MTHF}$ & $3.94^{\mathrm{A}}$ & 1.85 & $2 \cdot 47^{\mathrm{C}, \mathrm{B}}$ & 1.61 & $2 \cdot 17^{\mathrm{b}, \mathrm{C}}$ & 0.76 & $3.47^{\mathrm{b}}$ & 0.96 \\
\hline & 5-MTHF & 3.48 & 1.30 & $3 \cdot 53^{a, b}$ & 1.30 & $3.44^{\mathrm{a}}$ & 1.24 & $4 \cdot 75^{\mathrm{a}}$ & 1.62 \\
\hline & Placebo & $3 \cdot 76$ & 1.88 & $3 \cdot 71^{\mathrm{a}}$ & 1.58 & $3.59^{a}$ & 1.37 & $5 \cdot 14^{a}$ & 1.77 \\
\hline \multirow[t]{4}{*}{$\sum n-6: \sum n-3$} & FO & $12 \cdot 16^{\mathrm{A}}$ & $7 \cdot 88$ & $7 \cdot 93^{\mathrm{a}, \mathrm{b}, \mathrm{B}}$ & 4.47 & $6.94^{\mathrm{b}, \mathrm{c}, \mathrm{B}}$ & 4.02 & $5 \cdot 43^{\mathrm{b}}$ & 2.28 \\
\hline & $\mathrm{FO}+5-\mathrm{MTHF}$ & $10 \cdot 01^{A}$ & $6 \cdot 17$ & $7 \cdot 27^{\mathrm{b}, \mathrm{A}}$ & 6.76 & $6 \cdot 00^{\mathrm{c}, \mathrm{B}}$ & 2.98 & $5 \cdot 18^{\mathrm{b}}$ & 1.44 \\
\hline & 5-MTHF & $8 \cdot 62^{\mathrm{B}}$ & $4 \cdot 13$ & $9.68^{a, A}$ & 9.15 & $8 \cdot 10^{\mathrm{a}, \mathrm{b}, \mathrm{B}}$ & 3.89 & $7 \cdot 09^{a}$ & 2.60 \\
\hline & Placebo & $8 \cdot 70$ & 4.57 & $8 \cdot 77^{\mathrm{a}, \mathrm{b}}$ & 6.67 & $9 \cdot 00^{\mathrm{a}}$ & 6.59 & $7 \cdot 00^{\mathrm{a}}$ & 2.50 \\
\hline
\end{tabular}

FO, fish oil; 5-MTHF, 5-methyltetrahydrofoate; AA, arachidonic acid.

$\mathrm{a}, \mathrm{b}, \mathrm{c}$ Mean values within a column with unlike superscript letters were significantly different between the intervention groups $(P<0.05)$.

$\mathrm{A}, \mathrm{B}, \mathrm{C}$ Mean values within a row with unlike superscript letters were significantly different between the pregnancy time points $(P<0.05)$.

${ }^{*} n 36$.

$\dagger n 31$.

$\ddagger n 31$.

$\S n 30$.

intervention groups. The increments observed in the FOsupplemented groups (FO: 3.54 (SD 2.63) and FO + 5-MTHF: 3.87 (SD 2.46)) were significantly higher than those observed in the not FO-supplemented groups (5-MTHF: 1.44 (SD 1.75) and placebo: $1 \cdot 15(\mathrm{SD} 2 \cdot 04))(P<0 \cdot 001)$. DHA levels significantly increased between the 20th and 30th week of pregnancy in plasma PL and erythrocyte PC but no increment was observed between the 30th week of pregnancy and delivery. In contrast, DHA levels in erythrocyte PE continued to significantly rise between the 30th week of gestation and delivery (Figs. 1 and 2).

The degree of these changes was different in the various lipid classes, with higher percentage changes in erythrocyte PE (FO: $+67 \cdot 8 \%$ and FO + 5-MTHF: $+71 \cdot 8 \%$ ) and PC (FO: $+77 \cdot 6 \%$ and $\mathrm{FO}+5$-MTHF: $67 \cdot 1 \%)$ than in plasma PL (FO: $+28.4 \%$ and FO + 5-MTHF: $+28.6 \%)(P<0.001)$.
No differences in maternal or fetal fatty acid levels in both plasma or erythrocyte PL were observed between the groups supplemented with 5-MTHF and the groups that did not receive 5-MTHF supplements (Tables 2-4).

\section{Correlations between fatty acid relative levels (percentage of total fatty acids) in plasma and erythrocyte phospholipids}

Significant correlations were observed between DHA levels in maternal plasma and their levels in erythrocytes (PC and PE). Correlation coefficients in the FO-supplemented groups did not differ from those observed in the not FO-supplemented groups ( $P>0 \cdot 05$; Figs. 1 and 2; Table S2, available online).

DHA levels in cord plasma significantly correlated with their levels in cord erythrocytes. Correlation coefficients for the 
Table 4. Relative fatty acid composition (wt\%) of maternal and neonatal erythrocyte phosphatidylethanolamine (Mean values and standard deviations)

\begin{tabular}{|c|c|c|c|c|c|c|c|c|c|}
\hline & & \multicolumn{2}{|c|}{ Week 20} & \multicolumn{2}{|c|}{ Week 30} & \multicolumn{2}{|c|}{ Delivery } & \multicolumn{2}{|c|}{ Newborn } \\
\hline & & Mean & SD & Mean & SD & Mean & SD & Mean & SD \\
\hline \multirow[t]{4}{*}{$18: 2 n-6$} & $\mathrm{FO}^{*}$ & $6 \cdot 22^{A}$ & $1 \cdot 17$ & $5 \cdot 75^{\mathrm{b}, \mathrm{B}}$ & 1.09 & $5.99^{\mathrm{A}, \mathrm{B}}$ & 1.37 & $2 \cdot 74$ & 0.90 \\
\hline & $\mathrm{FO}+5-\mathrm{MTHF} \dagger$ & $6 \cdot 37^{\mathrm{A}}$ & $1 \cdot 13$ & $5 \cdot 86^{a, b, B}$ & 0.93 & $5 \cdot 90^{\mathrm{B}}$ & 0.94 & $2 \cdot 78$ & 0.67 \\
\hline & 5-MTHF $\ddagger$ & 6.04 & 0.98 & $6 \cdot 12^{\mathrm{a}}$ & 0.93 & $6 \cdot 21$ & 1.05 & $2 \cdot 72$ & 0.60 \\
\hline & Placebo§ & 5.97 & 0.95 & $6 \cdot 15^{a, b}$ & 0.90 & $6 \cdot 12$ & $1 \cdot 13$ & $2 \cdot 60$ & 0.83 \\
\hline \multirow[t]{4}{*}{$18: 3 n-3$} & FO & 0.21 & $0 \cdot 19$ & 0.19 & 0.16 & 0.21 & 0.20 & 0.14 & 0.18 \\
\hline & $\mathrm{FO}+5-\mathrm{MTHF}$ & 0.28 & 0.26 & 0.26 & 0.20 & 0.24 & 0.23 & 0.21 & 0.30 \\
\hline & 5-MTHF & 0.23 & 0.24 & 0.25 & 0.24 & 0.22 & 0.16 & 0.20 & 0.24 \\
\hline & Placebo & 0.20 & 0.17 & 0.22 & 0.21 & 0.23 & 0.21 & 0.12 & 0.12 \\
\hline \multirow[t]{4}{*}{$20: 4 n-6$} & FO & $20.36^{A}$ & $5 \cdot 34$ & $19 \cdot 20^{\mathrm{a}, \mathrm{b}, \mathrm{A}, \mathrm{B}}$ & $4 \cdot 14$ & $19 \cdot 19^{a, b, B}$ & 4.23 & $24 \cdot 30$ & 6.02 \\
\hline & $\mathrm{FO}+5-\mathrm{MTHF}$ & $19 \cdot 49^{\mathrm{A}}$ & $4 \cdot 86$ & $18.04^{\mathrm{a}, \mathrm{b}, \mathrm{B}}$ & 4.04 & $18 \cdot 13^{\mathrm{b}, \mathrm{B}}$ & 3.32 & 23.85 & $4 \cdot 18$ \\
\hline & 5-MTHF & $18 \cdot 82^{\mathrm{A}}$ & 4.97 & $17 \cdot 31^{\mathrm{b}, \mathrm{B}}$ & 4.34 & $18 \cdot 97^{\mathrm{a}, \mathrm{b}, \mathrm{A}, \mathrm{B}}$ & 3.59 & 24.35 & $5 \cdot 14$ \\
\hline & Placebo & $19 \cdot 65$ & 4.83 & $19 \cdot 64^{a}$ & 4.54 & $20 \cdot 09^{a}$ & 3.66 & $25 \cdot 19$ & 5.89 \\
\hline \multirow[t]{4}{*}{$22: 6 n-3$} & FO & $5 \cdot 22^{C}$ & 1.76 & $7 \cdot 20^{\mathrm{a}, \mathrm{b}, \mathrm{B}}$ & 2.33 & $8.76^{\mathrm{a}, \mathrm{A}}$ & 2.65 & $8 \cdot 64^{a, b}$ & 2.41 \\
\hline & $\mathrm{FO}+5-\mathrm{MTHF}$ & $5 \cdot 47^{C}$ & 1.80 & $7 \cdot 88^{\mathrm{a}, \mathrm{B}}$ & 2.73 & $9 \cdot 40^{\mathrm{a}, \mathrm{A}}$ & 2.65 & $9 \cdot 39^{\mathrm{a}}$ & 2.53 \\
\hline & 5-MTHF & $5 \cdot 43^{\mathrm{B}}$ & $2 \cdot 15$ & $5 \cdot 60^{\mathrm{c}, \mathrm{B}}$ & $2 \cdot 12$ & $6 \cdot 89^{\mathrm{b}, \mathrm{A}}$ & $2 \cdot 21$ & $7.76^{\mathrm{b}}$ & 1.90 \\
\hline & Placebo & $5 \cdot 42^{\mathrm{B}}$ & 1.99 & $5.94^{\mathrm{b}, \mathrm{c}, \mathrm{A}, \mathrm{B}}$ & 1.91 & $6 \cdot 54^{\mathrm{b}, \mathrm{A}}$ & 1.81 & $7.57^{\mathrm{b}}$ & 1.91 \\
\hline \multirow[t]{4}{*}{$\Sigma$ SFA } & FO & 36.43 & $5 \cdot 27$ & $36 \cdot 83$ & $5 \cdot 15$ & $35 \cdot 15$ & $4 \cdot 11$ & 39.04 & 6.06 \\
\hline & $\mathrm{FO}+5-\mathrm{MTHF}$ & $37 \cdot 34^{\mathrm{A}}$ & $6 \cdot 36$ & $35.91^{\mathrm{A}, \mathrm{B}}$ & 5.04 & $34.56^{\mathrm{B}}$ & 3.95 & 38.35 & 4.55 \\
\hline & 5-MTHF & $37.02^{A, B}$ & $5 \cdot 36$ & $38.07^{A}$ & $5 \cdot 67$ & $35 \cdot 35^{\mathrm{B}}$ & 4.62 & 38.95 & 5.39 \\
\hline & Placebo & 37.56 & $6 \cdot 12$ & $36 \cdot 18$ & 4.74 & 35.42 & 3.94 & 39.31 & $6 \cdot 11$ \\
\hline \multirow[t]{4}{*}{ ¿MUFA } & FO & $24 \cdot 15$ & 2.99 & $23 \cdot 36$ & 2.53 & $23 \cdot 20$ & $2 \cdot 19$ & $17 \cdot 62$ & $2 \cdot 35$ \\
\hline & $\mathrm{FO}+5-\mathrm{MTHF}$ & $23 \cdot 72$ & $2 \cdot 87$ & 24.62 & 3.29 & 23.98 & 3.02 & $17 \cdot 62$ & 2.51 \\
\hline & 5-MTHF & $24 \cdot 76$ & 3.07 & $25 \cdot 02$ & 3.74 & $24 \cdot 61$ & 3.06 & 17.87 & $2 \cdot 25$ \\
\hline & Placebo & $23 \cdot 87$ & 3.02 & 24.48 & 2.99 & $24 \cdot 19$ & 2.56 & $17 \cdot 61$ & 2.04 \\
\hline \multirow{4}{*}{ ¿PUFA } & FO & 36.92 & 7.09 & 37.51 & $6 \cdot 27$ & 39.55 & 5.56 & $41 \cdot 12$ & 7.44 \\
\hline & $\mathrm{FO}+5-\mathrm{MTHF}$ & $36 \cdot 46^{B}$ & $7 \cdot 13$ & $37 \cdot 23^{A, B}$ & 7.39 & $39 \cdot 31^{\mathrm{A}}$ & 5.69 & 41.69 & 5.68 \\
\hline & 5-MTHF & $35.54^{\mathrm{A}, \mathrm{B}}$ & $7 \cdot 37$ & $34 \cdot 16^{\mathrm{B}}$ & 6.81 & $37.68^{\mathrm{A}}$ & 5.65 & $40 \cdot 50$ & 6.56 \\
\hline & Placebo & 36.02 & 7.00 & 37.03 & 6.69 & $38 \cdot 14$ & 5.02 & 40.49 & $7 \cdot 31$ \\
\hline \multirow{4}{*}{ AA:DHA } & FO & $4 \cdot 17^{A}$ & 1.23 & $2.90^{\mathrm{b}, \mathrm{B}}$ & 1.02 & $2 \cdot 37^{\mathrm{b}, \mathrm{C}}$ & 0.80 & $2 \cdot 95^{\mathrm{a}, \mathrm{b}}$ & 0.79 \\
\hline & $\mathrm{FO}+5-\mathrm{MTHF}$ & $3.75^{\mathrm{A}}$ & 0.88 & $2 \cdot 47^{\mathrm{b}, \mathrm{B}}$ & 0.63 & $2 \cdot 04^{\mathrm{b}, \mathrm{C}}$ & 0.56 & $2 \cdot 73^{\mathrm{b}}$ & 0.87 \\
\hline & 5-MTHF & $3.83^{A}$ & $1 \cdot 17$ & $3 \cdot 38^{\mathrm{a}, \mathrm{A}}$ & 1.03 & $2 \cdot 95^{\mathrm{a}, \mathrm{B}}$ & 0.81 & $3 \cdot 26^{\mathrm{a}}$ & 0.81 \\
\hline & Placebo & $3.96^{\mathrm{A}}$ & $1 \cdot 17$ & $3 \cdot 51^{\mathrm{a}, \mathrm{B}}$ & 0.84 & $3 \cdot 23^{\mathrm{a}, \mathrm{B}}$ & 0.87 & $3.45^{\mathrm{a}}$ & 0.82 \\
\hline \multirow[t]{4}{*}{$\sum n-6: \sum n-3$} & FO & $3.65^{\mathrm{A}}$ & 0.85 & $2 \cdot 70^{\mathrm{b}, \mathrm{B}}$ & 0.75 & $2 \cdot 34^{\mathrm{b}, \mathrm{C}}$ & 0.67 & $2.95^{\mathrm{b}, \mathrm{c}}$ & 0.75 \\
\hline & $\mathrm{FO}+5-\mathrm{MTHF}$ & $3.46^{\mathrm{A}}$ & 0.83 & $2.45^{\mathrm{b}, \mathrm{B}}$ & 0.61 & $2 \cdot 06^{\mathrm{b}, \mathrm{C}}$ & 0.52 & $2 \cdot 72^{\mathrm{c}}$ & 0.65 \\
\hline & 5-MTHF & $3.49^{\mathrm{A}}$ & 1.04 & $3 \cdot 17^{\mathrm{a}, \mathrm{A}}$ & 0.88 & $2 \cdot 79^{\mathrm{a}, \mathrm{B}}$ & 0.71 & $3 \cdot 2^{a, b}$ & 0.73 \\
\hline & Placebo & $3.49^{\mathrm{A}}$ & 0.85 & $3 \cdot 27^{\mathrm{a}, \mathrm{A}, \mathrm{B}}$ & 0.79 & $3.04^{\mathrm{a}, \mathrm{B}}$ & 0.77 & $3 \cdot 40^{\mathrm{a}}$ & 0.67 \\
\hline
\end{tabular}

FO, fish oil; 5-MTHF, 5-methyltetrahydrofoate; AA, arachidonic acid.

$\mathrm{a}, \mathrm{b}, \mathrm{c}$ Mean values within a column with unlike superscript letters were significantly different between the intervention groups $(P<0 \cdot 05)$

$\mathrm{A}, \mathrm{B}, \mathrm{C}$ Mean values within a row with unlike superscript letters were significantly different between the pregnancy time points $(P<0.05)$.

${ }^{*} n 46$.

$\dagger n 42$.

$\ddagger n 33$.

$\S n 39$.

association were $r 0.354(P<0.001)$ and $r 0.348(P<0.001)$ for erythrocyte $\mathrm{PC}$ and $\mathrm{PE}$, respectively.

\section{Correlations between maternal and neonatal fatty acid} relative levels in plasma and erythrocyte phospholipids at delivery

DHA and arachidonic acid (AA) percentage levels in cord blood correlated significantly with fatty acid levels in maternal blood at delivery in both plasma and erythrocyte PL (Fig. 3). Correlation coefficients were higher in erythrocyte PE compared with those observed in plasma or erythrocyte PC $(P<0.05$; Table S3, available online).

\section{Discussion}

It is well known from interventional studies that mothers consuming $n$-3 LC-PUFA supplements have higher $n$-3 LC-PUFA levels in both plasma and erythrocytes during pregnancy and at delivery ${ }^{(3,7-9,15,19)}$. Higher levels of $n$-3 LC-PUFA have also been reported in umbilical cord plasma and erythrocyte PL in supplemented mothers ${ }^{(15,19,20)}$. Furthermore, DHA levels in cord blood plasma and erythrocytes have been demonstrated to correlate with maternal blood DHA levels ${ }^{(3,6,21,22)}$. In agreement with previous interventional studies, $\mathrm{FO}^{(3,7-9,15,19)}$ supplementation in the second half of pregnancy significantly increased DHA levels in maternal and umbilical plasma and erythrocyte PL. Supplementation did not significantly modify AA levels in umbilical erythrocyte membrane PL but AA levels in umbilical plasma PL were significantly lower in the FO-supplemented groups. Supplementation with $500 \mathrm{mg} / \mathrm{d}$ of DHA in populations with a mean daily DHA intake above the recommended $200 \mathrm{mg} / \mathrm{d}^{(1,23)}$ may lower AA in the fetus. The supplementation with a AA-DHA blend has proven to increase DHA levels without decreasing 

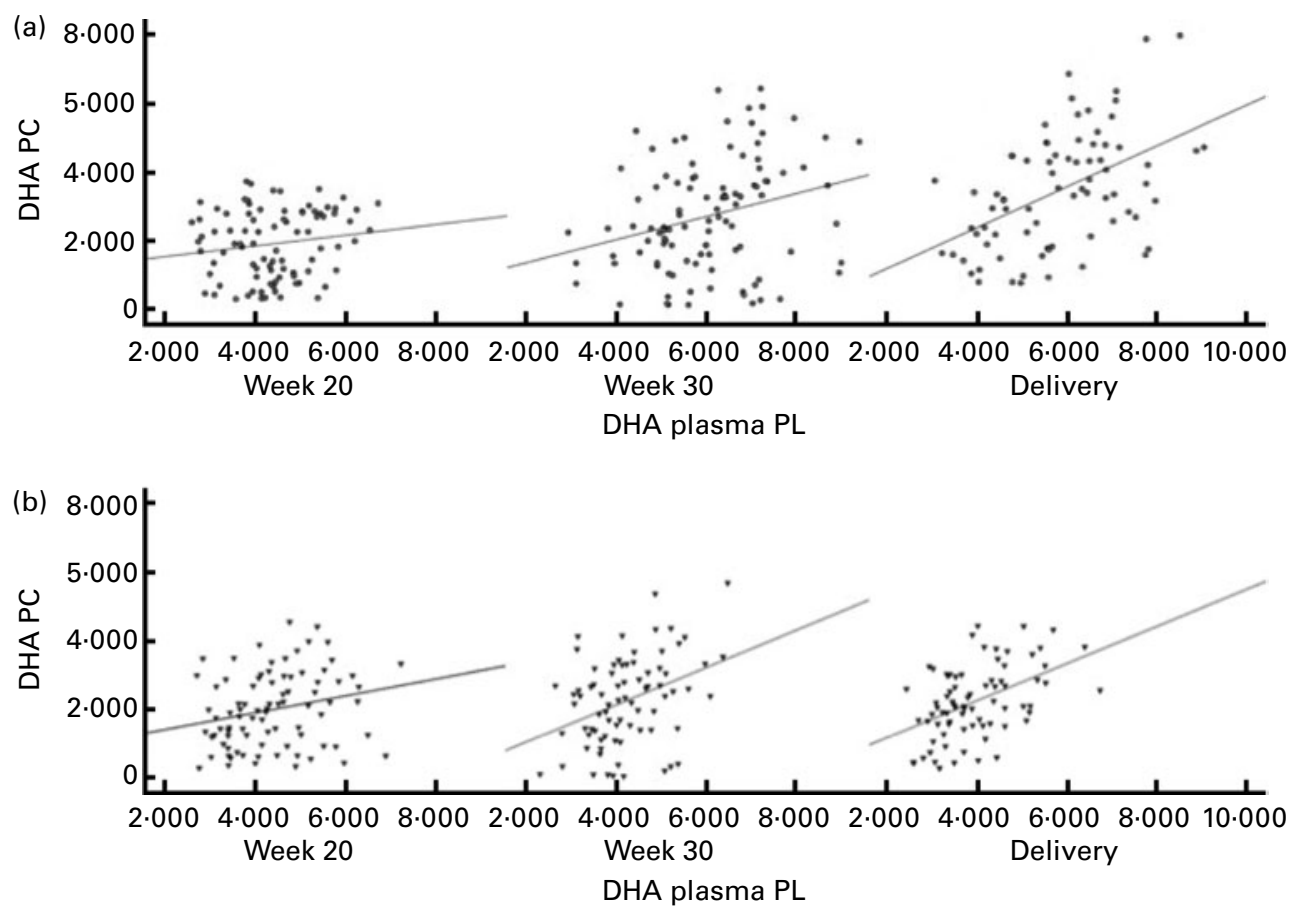

Fig. 1. Scattered data graph of DHA percentage concentrations in plasma and erythrocyte membrane phosphatidylcholine (PC) during the course of pregnancy in (a) the fish oil (FO)-supplemented (FO and FO + 5-methyltetrehydrofolate) and (b) not supplemented (placebo and 5-methyltetrahydrofolate) groups. (a) Week 20: $R$ 0.154, $P=0.152$; week 30: $R$ 0.275, $P=0.005$; delivery: $R$ 0.485, $P<0.001$. (b) Week 20: $R$ 0.234, $P=0.031$; week 30: $R$ 0.372, $P=0.001$; delivery: $R$ 0.467, $P<0.001$. PL, phospholipid; DHA PC, DHA levels in erythrocyte membrane PC.

the levels of AA and may be a better way of avoiding the AA fall following $n$-3 LC-PUFA supplementation ${ }^{(9)}$.

Human fatty acid status depends only partially on dietary intake. Genetic and metabolic factors, as well as lifestyle determinants, may affect fatty acid concentrations in human tissues. Furthermore, pregnancy may modify plasma and erythrocyte fatty acid concentrations as a result of the occurring metabolic changes. Several studies have been conducted
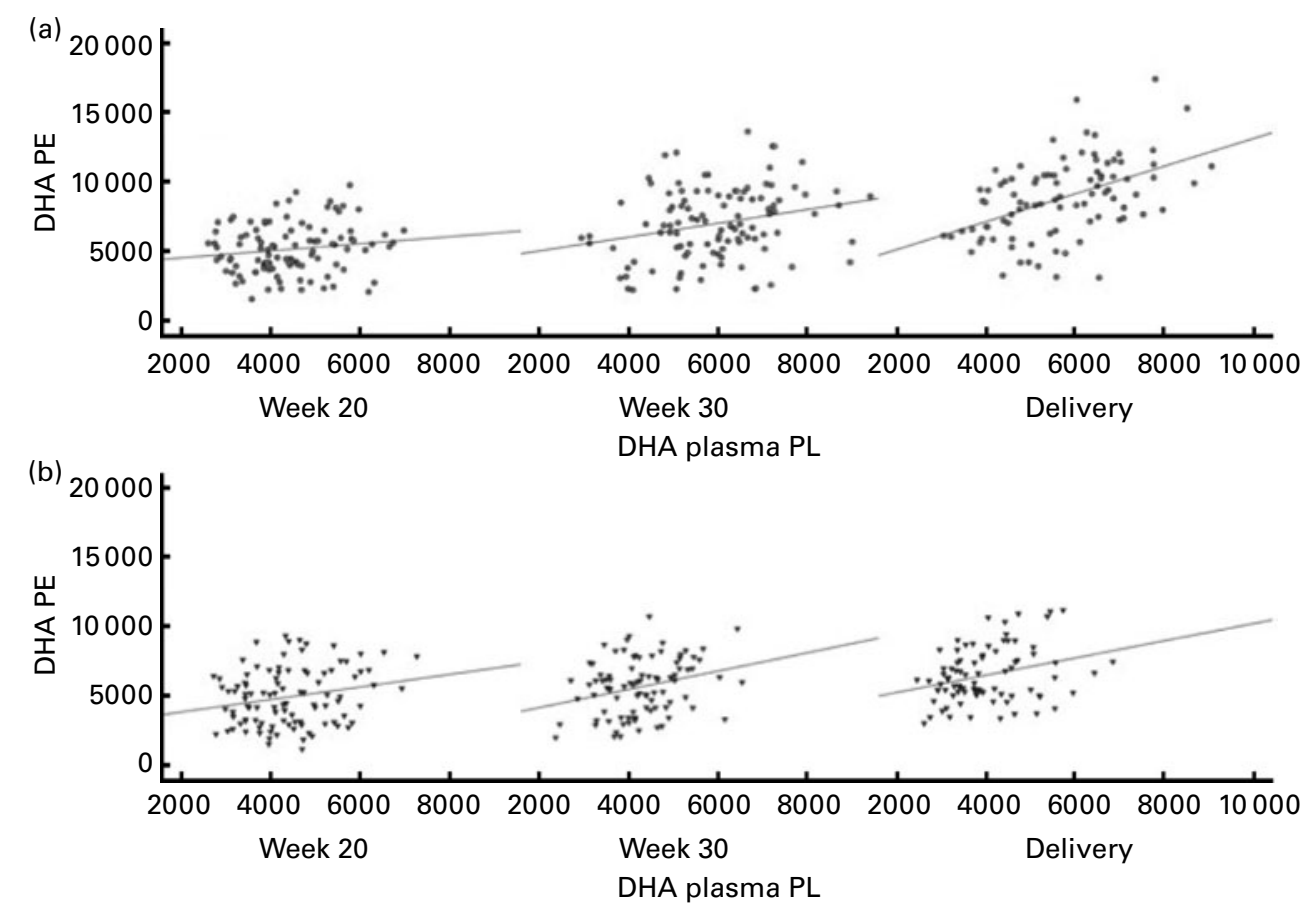

Fig. 2. Scattered data graph of $\mathrm{DHA}$ relative concentrations in plasma and erythrocyte membrane phosphatidylethanolamine (PE) during the course of pregnancy in (a) the fish oil (FO)-supplemented (FO and FO + 5-methyltetrahydrofolate) and (b) not supplemented (placebo and 5-methyltetrahydrofolate) groups. (a) Week 20: $R$ 0.148, $P=0.133$; week 30: $R 0.252, P=0.008$; delivery: $R 0.473, P<0.001$. (b) Week $20: R 0.219, P=0.028$; week 30 : $R 0.276, P=0.008$; delivery: $R$ 0.292, $P=0.007$. PL, phospholipid; DHA PE, DHA levels in erythrocyte membrane PE. 
(a)

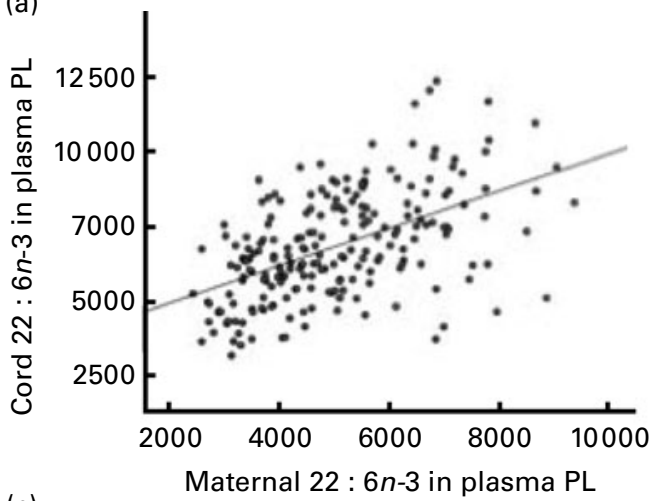

(c)

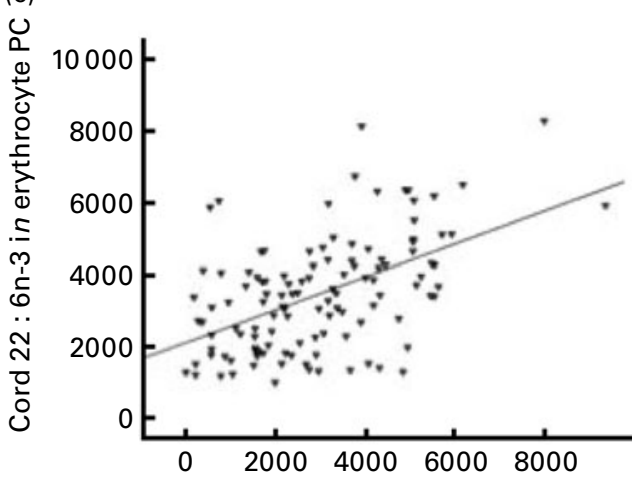

Maternal 22 : $6 n-3$ in erythrocyte PC

(e)

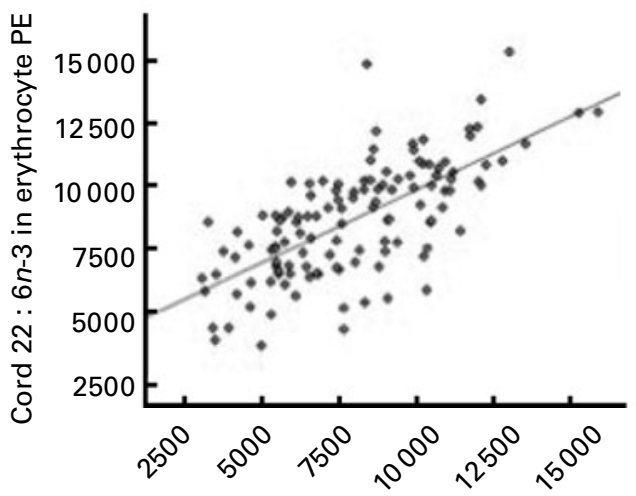

Maternal $22: 6 n-3$ in erythrocyte PE (b)

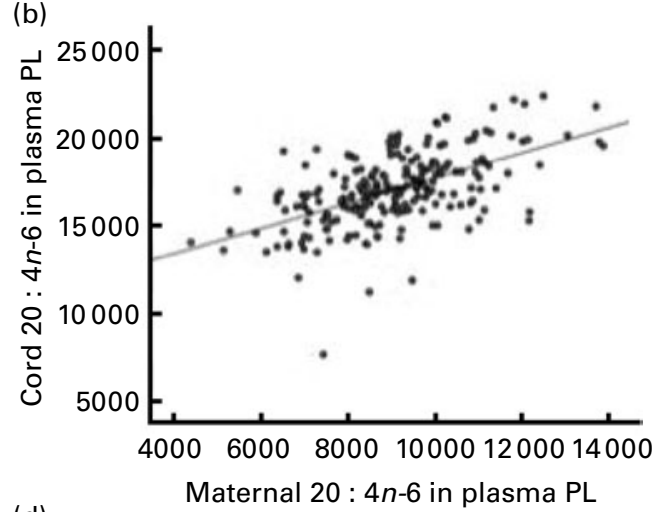

(d)



Maternal 20 : $4 n-6$ in erythrocyte PC

(f)

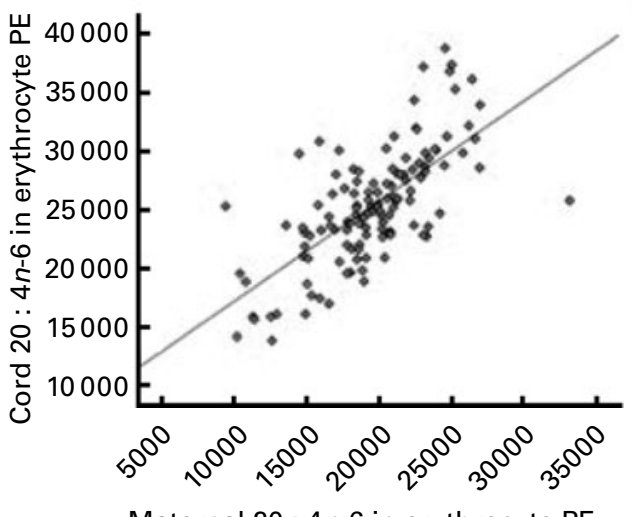

Fig. 3. Linear regression models for the relationship between maternal $(a, c, e)$ DHA and $(b, d, f)$ arachidonic acid levels expressed as a percentage of total fatty acids $(w t \%)$ at delivery and their relative levels in cord plasma and erythrocyte membrane (c, d) phosphatidylcholine (PC) and (e, f) phosphatidylethanolamine (PE). (a) $R 0.522, P<0.001$; (b) $R 0.559, P<0.001$; (c) $R 0.516, P<0.001$; (d) $R 0.404, P<0.001$; (e) $R 0.686, P<0.001$; (f) $R 0.690, P<0.001$. PL, phospholipid.

in order to establish the most confident compartment for the assessment of the LC-PUFA status of individuals. A revised meta-analysis of intervention studies concluded that both plasma and erythrocyte PL appear to be good markers for DHA status in humans, but special consideration should be given to particular population subgroups ${ }^{(24)}$. Only a few studies have evaluated the influence of $n-3$ PUFA supplements during the course of pregnancy in both erythrocytes and plasma in the same population ${ }^{(8,9)}$.

Studies on the kinetics of fatty acid incorporation into plasma PL and erythrocytes after DHA supplementation have shown a dose-dependent rapid increase of DHA in plasma
PL with a subsequent steady-state concentration 1 month after the start of supplementation. Erythrocytes follow a similar pattern but it takes $4-6$ months to reach equilibrium ${ }^{(25-27)}$. In addition, most of the $\mathrm{PE}$ in erythrocyte membranes is located in the inner layer, whereas PC is in the outer layer and can therefore interact more easily with circulating plasma lipids. Thus, PC reflects more directly the fatty acid composition of plasma PL, whereas the fatty acid composition of PE appears to be more dependent on a selective incorporation of LC-PUFA into PE. A study on DHA supplementation in healthy adult vegetarians showed strong correlations between erythrocyte PC and plasma PL fatty acid changes, while 
erythrocyte PE and plasma PL fatty acid changes were less closely related ${ }^{(28)}$. After the first 10 weeks of supplementation in the present study, DHA levels in maternal plasma and erythrocyte PC may have risen to their steady-state levels and consequently do not continue to increase in the last weeks of pregnancy. DHA in erythrocyte PE may need longer to reach the steady-state concentration and therefore continues to increase until delivery. Given the slow turnover of fatty acids in cell membranes, erythrocyte PL may be a more reliable measure of long-term fatty acid intake, whereas plasma PL appear more sensitive to short-term changes in the intake of LC-PUFA and may be more suitable to monitor the fatty acid status of individuals in intervention studies. Both plasma and erythrocyte fatty acids appear adequate to assess the fatty acid status in pregnant women and their neonates but the type of study is a major consideration when determining which body compartment reflects a better measure of fatty acid status.

It should be taken into account that studies on the kinetics of incorporation of fatty acids in plasma and erythrocyte PL have not been conducted in pregnant women. Most studies conducted in pregnancy have reported a similar evolution pattern of fatty acids in both plasma and erythrocyte and reported the positive correlations between maternal plasma and erythrocyte fatty acid levels and fatty acid changes throughout gestation $^{(21,22,29)}$, which is consistent with the present results. In general, changes observed in erythrocyte membrane PL mirror those occurring in plasma PL but we observed that the degrees of DHA percentage changes were different in the various lipid fractions. Geppert et $a l .{ }^{(28)}$ in their study in healthy adult vegetarians reported that relative changes of DHA 8 weeks after supplementation were greater in plasma and erythrocyte PC than in erythrocyte total lipids and erythrocyte $\mathrm{PE}$, which contrast with the present finding of greater changes in erythrocyte PE and PC compared with plasma PL. Vlaardingerbroek \& Hornstra ${ }^{(22)}$ observed in their study that the amount of most fatty acids continued to increase in erythrocytes after 32 week of pregnancy while the amounts in plasma hardly increased or even decreased, which is consistent with the present findings. These authors have suggested that the increment observed in erythrocyte PL could be explained by the replacement during pregnancy of erythrocyte PL classes with one ester-linked fatty acid moiety by PL classes carrying two ester-linked fatty acids, such as $\mathrm{PC}$ and $\mathrm{PE}^{(30)}$. As a result, plasma lipids could be more readily adsorbed to the erythrocyte membrane, which could explain the higher percentage increase of DHA in erythrocyte PC and PE. There is evidence suggesting that erythrocytes play an important role in the transport of DHA into the fetus ${ }^{(31)}$; the increment of PE and PC in erythrocyte membrane may be an adaptation mechanism during pregnancy to increase the transfer of LC-PUFA. However, further research is needed to elucidate the mechanism of how erythrocytes provide LC-PUFA to the fetus.

We observed positive significant correlations between plasma and erythrocyte DHA levels, which is consistent with previously reported data ${ }^{(21,22,29)}$. Our correlation coefficients were considerably lower than those reported in non-pregnant individuals $^{(28)}$. Fatty acids bind to TAG in high proportion during pregnancy ${ }^{(32)}$, and the fact that we did not consider the LC-PUFA fraction bound to TAG in the present study could explain our lower correlation coefficients.

Correlation coefficients between maternal and cord fatty acid relative levels at delivery were significantly higher in erythrocyte PE compared with the correlation coefficients in plasma PL. Plasma levels of lipoproteins in cord serum are low, with HDL being the major lipoprotein and LDL and VLDL present in low and very low concentrations $(33,34)$ Thus, in agreement with previously reported data $^{(31)}$, it could be hypothesised that erythrocyte membrane PL are important carriers of LC-PUFA in fetal blood, which could explain the higher correlation coefficients between maternal and fetal fatty acid percentage levels in erythrocyte membrane PL. These results suggest that erythrocyte PL seem to be a more reliable biomarker for the prediction of fetal/neonatal fatty acid status on the basis of maternal fatty acid levels.

Both plasma and erythrocyte fatty acid levels seem to be suitable to assess maternal fatty acid status. More information on the physiological changes taking place in the erythrocyte membrane during gestation, as well as further knowledge about fetal metabolism and the kinetics of fatty acid accretion in fetal tissues, is needed. Nevertheless, fatty acid levels in erythrocyte PL seem to be a more reliable biomarker compared with those in plasma PL to predict the fetal/ neonatal fatty acid status on the basis of maternal blood fatty acid levels.

\section{Supplementary material}

To view supplementary material for this article, please visit http://dx.doi.org/10.1017/S0007114512003716.

\section{Acknowledgements}

The authors thank all participating women for their collaboration and all colleagues in the study centres for their support. This study was carried out with partial financial support from the Commission of the European Communities, specific RTD Programme 'Quality of Life and Management of Living Resources', within the 5th Framework Programme (contract no. QLK1-CT-1999-00888 NUHEAL (Nutraceuticals for a healthier life)). This paper does not necessarily reflect the views of the Commission and in no way anticipates the future policy in this area. All authors have made substantive contributions to the study, and endorse the data and conclusions. B. V. K. is the recipient of a Freedom to Discover Award of the Bristol Myers Squibb Foundation, New York, NY, USA. The authors' contributions are as follows: M. V. E.-M. wrote the paper; C. C., B. V. K. and T. D. designed and coordinated the research; C. C. supervised the writing; T. D. was responsible for the erythrocyte membrane fatty acid analyses; M. C. R.-T. and A. G. were responsible for the fatty acid analyses in plasma PL; H. D. conducted the research; M. T. M. analysed the data. All authors certify that there is no actual or potential conflict of interest in relation to this article. 


\section{References}

1. Koletzko B, Lien E, Agostoni C, et al. (2008) The roles of long-chain polyunsaturated fatty acids in pregnancy, lactation and infancy: review of current knowledge and consensus recommendations. J Perinat Med 36, 5-14.

2. Helland IB, Saugstad OD, Saarem K, et al. (2006) Supplementation of $n-3$ fatty acids during pregnancy and lactation reduces maternal plasma lipid levels and provides DHA to the infants. J Matern Fetal Neonatal Med 19, 397-406.

3. Dunstan JA, Mori TA, Barden A, et al. (2004) Effects of $n-3$ polyunsaturated fatty acid supplementation in pregnancy on maternal and fetal erythrocyte fatty acid composition. Eur J Clin Nutr 58, 429-437.

4. Bergmann RL, Haschke-Becher E, Klassen-Wigger $\mathrm{P}$, et al. (2008) Supplementation with $200 \mathrm{mg} /$ day docosahexaenoic acid from mid-pregnancy through lactation improves the docosahexaenoic acid status of mothers with a habitually low fish intake and of their infants. Ann Nutr Metab $\mathbf{5 2}$, 157-166.

5. Innis SM \& Friesen RW (2008) Essential $n$-3 fatty acids in pregnant women and early visual acuity maturation in term infants. Am J Clin Nutr 87, 548-557.

6. Smuts CM, Borod E, Peeples JM, et al. (2003) High-DHA eggs: feasibility as a means to enhance circulating DHA in mother and infant. Lipids 38, 407-414.

7. Sanjurjo P, Ruiz-Sanz JI, Jimeno P, et al. (2004) Supplementation with docosahexaenoic acid in the last trimester of pregnancy: maternal-fetal biochemical findings. J Perinat Med 32, 132-136.

8. Montgomery C, Speake BK, Cameron A, et al. (2003) Maternal docosahexaenoic acid supplementation and fetal accretion. Br J Nutr 90, 135-145.

9. Otto SJ, van Houwelingen AC \& Hornstra G (2000) The effect of supplementation with docosahexaenoic and arachidonic acid derived from single cell oils on plasma and erythrocyte fatty acids of pregnant women in the second trimester. Prostaglandins Leukot Essent Fatty Acids 63, 323-328.

10. Baylin A \& Campos H (2006) The use of fatty acid biomarkers to reflect dietary intake. Curr Opin Lipidol $\mathbf{1 7}$ $22-27$.

11. Sun Q, Ma J, Campos H, et al. (2007) Comparison between plasma and erythrocyte fatty acid content as biomarkers of fatty acid intake in US women. Am J Clin Nutr 86, 74-81.

12. Umhau JC, Dauphinais KM, Patel SH, et al. (2006) The relationship between folate and docosahexaenoic acid in men. Eur J Clin Nutr 60, 352-357.

13. Sugiyama K, Kumazawa A, Zhou H, et al. (1998) Dietary methionine level affects linoleic acid metabolism through phosphatidylethanolamine N-methylation in rats. Lipids $\mathbf{3 3}$ 235-242.

14. Pita ML \& Delgado MJ (2000) Folate administration increases $n-3$ polyunsaturated fatty acids in rat plasma and tissue lipids. Thromb Haemost 84, 420-423.

15. Krauss-Etschmann S, Shadid R, Campoy C, et al. (2007) Effects of fish-oil and folate supplementation of pregnant women on maternal and fetal plasma concentrations of docosahexaenoic acid and eicosapentaenoic acid: a European randomized multicenter trial. Am J Clin Nutr 85, 1392-1400.

16. Kolarovic L \& Fournier NC (1986) A comparison of extraction methods for the isolation of phospholipids from biological sources. Anal Biochem 156, 244-250.

17. Agren JJ, Julkunen A \& Penttila I (1992) Rapid separation of serum lipids for fatty acid analysis by a single aminopropyl column. J Lipid Res 33, 1871-1876.
18. Lepage G \& Roy CC (1986) Direct transesterification of all classes of lipids in a one-step reaction. J Lipid Res $\mathbf{2 7}$, $114-120$.

19. Helland IB, Saugstad OD, Smith L, et al. (2001) Similar effects on infants of $n-3$ and $n-6$ fatty acids supplementation to pregnant and lactating women. Pediatrics 108, E82.

20. Barden AE, Dunstan JA, Beilin LJ, et al. (2006) n-3 Fatty acid supplementation during pregnancy in women with allergic disease: effects on blood pressure, and maternal and fetal lipids. Clin Sci (Lond) 111, 289-294.

21. Matorras R, Perteagudo L, Sanjurjo P, et al. (1999) Intake of long chain $\mathrm{w} 3$ polyunsaturated fatty acids during pregnancy and the influence of levels in the mother on newborn levels. Eur J Obstet Gynecol Reprod Biol 83, 179-184.

22. Vlaardingerbroek H \& Hornstra G (2004) Essential fatty acids in erythrocyte phospholipids during pregnancy and at delivery in mothers and their neonates: comparison with plasma phospholipids. Prostaglandins Leukot Essent Fatty Acids $\mathbf{7 1}$, 363-374.

23. Franke C, Verwied-Jorky S, Campoy C, et al. (2008) Dietary intake of natural sources of docosahexaenoic acid and folate in pregnant women of three European cohorts. Ann Nutr Metab 53, 167-174.

24. Fekete K, Marosvolgyi T, Jakobik V, et al. (2009) Methods of assessment of $n-3$ long-chain polyunsaturated fatty acid status in humans: a systematic review. Am J Clin Nutr 89, 2070S-2084S

25. Katan MB, Deslypere JP, van Birgelen AP, et al. (1997) Kinetics of the incorporation of dietary fatty acids into serum cholesteryl esters, erythrocyte membranes, and adipose tissue: an 18-month controlled study. J Lipid Res 38, 2012-2022.

26. Cao J, Schwichtenberg KA, Hanson NQ, et al. (2006) Incorporation and clearance of omega- 3 fatty acids in erythrocyte membranes and plasma phospholipids. Clin Chem 52, 2265-2272.

27. Arterburn LM, Hall EB \& Oken H (2006) Distribution, interconversion, and dose response of $n-3$ fatty acids in humans. Am J Clin Nutr 83, Suppl. 6, 1467S-1476S.

28. Geppert J, Kraft V, Demmelmair H, et al. (2005) Docosahexaenoic acid supplementation in vegetarians effectively increases omega-3 index: a randomized trial. Lipids $\mathbf{4 0}$, $807-814$.

29. Courville AB, Keplinger MR, Judge MP, et al. (2009) Plasma or red blood cell phospholipids can be used to assess docosahexaenoic acid status in women during pregnancy. Nutr Res 29, 151-155.

30. Vaysse J, Dureuil M, Pilardeau P, et al. (1986) Plasma and erythrocyte lipid composition in normal pregnancy. Biol Res Pregnancy Perinatol 7, 89-92.

31. Ruyle M, Connor WE, Anderson GJ, et al. (1990) Placental transfer of essential fatty acids in humans: venous-arterial difference for docosahexaenoic acid in fetal umbilical erythrocytes. Proc Natl Acad Sci U S A 87, 7902-7906.

32. Herrera E (2002) Implications of dietary fatty acids during pregnancy on placental, fetal and postnatal development - a review. Placenta 23, S9-19.

33. Legras B, Durou MR, Ruelland A, et al. (1995) Serum lipid, apolipoprotein and lipoparticle levels in the human fetus. Prenat Diagn 15, 225-228.

34. Nagasaka H, Chiba H, Kikuta H, et al. (2002) Unique character and metabolism of high density lipoprotein (HDL) in fetus. Atherosclerosis 161, 215-223. 